\title{
Cannabis : \\ de nouvelles inquiétudes
}

Communiqué de I'Académie Nationale de Médecine - Commission VI (Addictions)

\section{RÉSUMÉ}

\section{Roger NORDMANN}

Membre de l'Académie Nationale

de Médecine,

Président de la Commission «Addictions».
L'usage régulier du cannabis par plus de 1200000 français suscite des inquiétudes en raison de ses effets délétères :

- forte dépendance psychique ;

- forte dépendance physique ;

- risque de son association avec l'alcool ;

- incitation à la consommation d'autres drogues.

Cet usage a incité l'Académie Nationale de Médecine à formuler un certain nombre de recommandations.

(C) AEOS / EDP Sciences 
L'Académie nationale de médecine a attiré dès 1995 l'attention sur les risques sanitaires et sociaux que peut entraîner la consommation de cannabis[1]. Elle a émis par la suite plusieurs communiqués exprimant son inquiétude face à la banalisation croissante de cette consommation[2-7], banalisation favorisée par une comparaison, source de confusion, avec les effets de l'alcool et du tabac[8]. Plusieurs ouvrages récents ont, de leur côté, décrit l'ensemble des conséquences délétères que le cannabis est à même $d$ 'induire, particulièrement en cas d'usage régulier ou intensif[9-12].

Ces diverses actions n'ont cependant pas limité la diffusion de la consommation de cannabis en France $[12,13]$ qui suscite, par son ampleur et sa gravité, de nouvelles et très vives inquiétudes.

Cette toxicomanie concerne en effet un million deux cent mille Français qui sont devenus des usagers réguliers (plus de neuf fois par mois), tandis que cinq cent mille d'entre eux sont des consommateurs quotidiens, et souvent plusieurs fois par jour[12]. Parmi les 27 Etats membres de l'Union Européenne, la France se situe en tête en ce qui concerne la consommation de cannabis [14]. Le développement de cette toxicomanie s'explique en partie par le fait que l'on trouve aisément sur l'Internet de nombreux sites commerciaux qui exercent une propagande scandaleuse en proposant des semences de cannabis, des conseils de culture avec milieux de culture, engrais et matériels divers, des recettes pour faire les «joints» avec du tabac et de la résine, des modèles de pipes pour fumer et notamment des pipes à eau.

\section{> Ce phénomène connaît, depuis peu, plusieurs facteurs de gravité supplémentaires}

- La précocité des premières consommations, or la détérioration psychique est d'autant plus rapide que l'usage est plus précoce[15] ;

- la diffusion de la pipe à eau, nouveau mode d'absorption particulièrement redoutable, car délivrant au cerveau, en un bref délai, des quantités importantes de tétrahydrocannabinol (THC), principe actif majeur du cannabis, suscitant d'emblée délire et hallucinations[13] ;

- I'accroissement fréquent de la teneur en THC dans les produits en circulation (marijuana, haschich), qui a doublé entre 1993 et 2004[11] et a été multipliée dans certains produits par 5, voire plus, depuis 40 ans ;

- il convient de rappeler l'exceptionnelle lipophilie du THC qui fait que, de toutes les drogues, il est la seule à se stocker, pour des jours ou des semaines, dans le cerveau, organe particulièrement riche en lipides.

La dépendance psychique est forte, à la mesure du nombre de ceux qui, l'ayant expérimenté, lui sont devenus assujettis (environ $20 \%$ d'entre eux). La dépendance physique, longtemps niée, est désormais avérée, mais les troubles sont différés d'une à deux semaines, après l'arrêt de toute consommation, compte tenu de la longue rémanence du THC dans l'organisme[16, 17]. Elle peut être révélée, de façon intense et instantanée, par l'administration d'un agent bloquant (antagoniste) des récepteurs CB1 auxquels se lie le THC[18].

\section{> D'autres dangers de cette drogue ont été récemment établis}

- Comme l'a démontré l'étude SAM (Stupéfiants et Accidents Mortels de la route)[19], le cannabis, par ses effets sédatifs (psycholeptiques) et enivrants, est à lui seul responsable de 250 morts par an; son association à l'alcool multiplie par 14 le risque d'accident mortel, et pourtant ces chiffres inquiétants sont minimisés par le seuil élevé du THC san- 
guin retenu dans cette étude, alors même que I'on sait bien que, lorsque le THC a quitté le sang, il est encore à l'œuvre dans le cerveau.

- Il est maintenant confirmé qu'une consommation importante de cannabis exerce des effets anxiogènes[20], induit des troubles dépressifs[21] et parfois des effets désinhibiteurs[22], conduisant à des prises de risques ou à des comportements violents[23]. Un syndrome amotivationnel[24] peut survenir, d'où résulte une perturbation des mécanismes éducatifs, encore aggravée par les troubles de la mémoire[25] et de l'attention.

- La décompensation d'états pré-schizophréniques, la survenue de novo d'une psychose dite cannabique et la résistance aux traitements antipsychotiques sont désormais bien démontrées[26].

\section{Recommandations}

Compte tenu de ces constats inquiétants, l'Académie Nationale de Médecine :

- demande avec force que les politiques de prévention et de communication sur le cannabis soient renforcées, sans aucune concession aux défenseurs du cannabis ;

- s'émeut de la libre installation de magasins fournissant tous les outils de la culture domestique du chanvre indien, avec la libre acquisition sur I'Internet des semences de variétés à très haute teneur en THC, ainsi que de pipes à eau, livrées à domicile par la Poste et invite les Pouvoirs Publics à une particulière vigilance dans ce domaine ;

- demande que, dans de larges plages de l'emploi du temps de l'enseignement primaire,
- Parmi les autres méfaits de ce produit, il faut énumérer :

- l'incitation fréquente à la consommation d'autres drogues (alcool, tabac, héroïne) $[13,27]$;

- les effets cancérogènes dans les domaines O.R.L. et broncho-pulmonaires, au moins égaux à ceux du tabac[28, 29], ainsi que I'action délétère sur l'appareil respiratoire conduisant à la bronchite chronique obstructive[30] ;

- les risques d'atteintes cardio-vasculaires et de décès par infarctus du myocarde[31, 32] ;

- la modification du statut endocrinien avec baisse de la libido et diminution de la fertilité[33] ;

- la perturbation du déroulement de la grossesse, ainsi que du développement physique et neuropsychique du nourrisson[6, 34, 35].

des collèges, lycées, I.U.F.M., I.U.T., universités, écoles d'ingénieurs, grandes écoles, soient dispensées, par des professionnels de la santé formés à cette communication, des informations scientifiques de qualité et des mises en garde sur les toxicomanies en général et le cannabis en particulier ;

- demande que les stages d'information sur les dangers des drogues, auxquels devront se soumettre les contrevenants à l'usage du cannabis, s'inscrivent dans une communication maîtrisée, soigneusement élaborée, contrôlée et évaluée ;

- affirme à nouveau la nécessité de mesures de dépistage précoce et de prévention au cours d'une visite pré-conceptionnelle et du premier trimestre de la grossesse ; 
- préconise que, pour l'accès à certaines fonctions ou responsabilités "sensibles», on puisse, à l'exemple de ce qui est déjà le cas pour certaines professions, vérifier l'absence de cannabinoïdes dans l'urine, permettant ainsi de s'assurer que le candidat n'est pas dépendant du cannabis ;
- suggère la réalisation de contrôles sur les routes et dans les milieux professionnels où cela parait nécessaire, afin de s'assurer que les conducteurs ou certains professionnels ne sont pas sous l'emprise du cannabis ou d'autres stupéfiants susceptibles de les rendre dangereux pour autrui.

\section{Bibliographie}

1. Lechat $\mathrm{P}$, et Juillet $\mathrm{P}$. Sur le cannabis.

Bull Acad Nat Méd 1995; 179:1313.

2. Lechat $P$. Position de l'Académie nationale de médecine sur le cannabis. Bull Acad Nat Méd 1998; 182:1281-1283.

3. Nordmann $\mathrm{R}$, Juillet $\mathrm{P}$. Motifs récents d'inquiétude à propos du cannabis. Bull Acad Nat Méd 2001;185:1185-1186.

4. Nordmann R. Drogues illicites d'aujourd'hui et santé. Bull Acad Nat Méd 2002;186:571-572.

5. Nordmann R. A propos de l'usage des drogues illicites et notamment du cannabis. Bull Acad Nat Méd 2002;186:1321-1322.

6. Nordmann R. Consommation d'alcool, de tabac ou de cannabis au cours de la grossesse. Bull Acad Nat Méd 2004;188:519-521.

7. Tubiana M. Le cannabis.
In : Désamorcer le cannabis dès l'école.

Nordmann R. (Dir.), Lavoisier 2006:21-22.

8. Nordmann R Le cannabis est-il moins dangereux que I'alcool et le tabac ? Bull Acad Nat Méd 2003;187:1019-1020.

9. Reynaud M. Cannabis et santé. Flammarion Médecine Sciences 2004:194 p.

10. Plasait B. Cannabis. Scandaleuses complaisances F.X. de Guibert 2004:182 p.

11. Costentin J. Halte au cannabis. Odile Jacob 2006:264 p.

12. Costes J.M. (Dir.)

Cannabis, données essentielles. OFDT 2007:232 p. (www.ofdt.fr).

13. Costentin J. Données neurobiologiques sur le cannabis en 2006. In : Désamorcer le cannabis dès l'école. Nordmann R. (Dir.), Lavoisier 2006:31-64.

14. Observatoire européen des drogues et des toxicomanies Rapport annuel 2007 sur l'état du phénomène de la drogue en Europe. http://www.ofdt.fr/BDD/ publications/docs/efnxofna. pdf

15. Lynskey MT, Glowinsky $A L$, Todorov A.A et al. Major depressive disorder, suicidal ideation, and suicide attempt in twins discordant for cannabis dependance and early-onset cannabis use. Arch Gen Psychiatry 2004;61:1026-1032.

16. Reynaud M.

Réalités

de la dépendances au cannabis. In : Traité d'addictologie. Reynaud M. (Dir.), Médecine-Sciences, Flammarion 2006:477-482.

17. Budney AJ, Hughes JR, Moore BA, Vandrey R. Review of the validity and significance of cannabis withdrawal syndrome. Am J Psychiatry 2004;161:1967-1977

18. Beardsley PM, Martin BR. Effects of 
the cannabinoid CB1 receptor antagonist, SR141716A, after Delta (9) tetrahydrocannabinol withdrawal.

Eur J Pharmacol 2000;387: 47-53.

19. Laumon B, Gadekbeku B, Martin J.L, et le Groupe SAM. Stupéfiants et accidents mortels (étude SAM).

Observatoire Français des drogues et des Toxicomanies (OFDT). Octobre 2005.

20. Hayatbakhsh MR Najman JM, Jamrozik K, Mamun A.A, Alati R, Bor W. Cannabis and anxiety and depression in young adults: a large prospective study. J Am Acad Child Adolesc Psychiatry 2007;46:408-417.

21. Patton GC, Coffey $C$, Carlin JB, Degenhardt L, Lynskey M, Hall W. Cannabis use and mental health in young people: cohort study. B.M.J. 2002;325:1195-1198.

22. Friedman AS, Terras A, Glassman K. The differential dishinibition effect of marijuana on violent behavior: a comparison of this effect on a conventionnal, non-delinquent group versus a delinquent or deviant group. J Addict Dis 2003;22:63-78.

23. Niveau G, Dang C. Cannabis and violent crime.
Med Sci Law 2003;43:115-121.

24. Nicholson AN, Turner $C$, Stone BM, Robson PJ. Effect of

Delta-9-

tetrahydrocannabinol and cannabidiol on nocturnal sleep and early-morning behavior in young adults. J Clin Psychopharmacol 2004;24:305-313.

25. Fried P.A, Watkinson B, Gray R.

Neurocognitive consequences of marijuana a comparison with pre-drug performance.

Neurotoxicol Teratol 2005;27:231-239.

26. Moore TH, Zammit $\mathrm{S}$, Lingford-Hughes A. et al. Cannabis use and risk of psychotic or affective mental health outcomes:

a systematic review. Lancet 2007;370:319-328.

27. Mechoulam R, Parker L. Cannabis and alcohol a close friendship. Trends Pharmacol Sci 2003;24:266-268

28. Hashibe M, Straif $K$, Tashkin DF, Morgenstern $\mathrm{H}$, Greenland S, Zhang ZF. Epidemiologic review of marijuana use and cancer risk. Alcohol 2005;35:265-275.

29. Voirin N, Berthiller J, Benhaim-Luzon V. et al. Risk of lung cancer and past use of cannabis in Tunisia.

J Thorac Oncol 2006;1:

577-579.

30. Aldington $S$, Williams $M$, Nowitz M. et al.

Effects of cannabis on pulmonary structure, function and symptoms. Thorax 2007;62:1058-1063.

31. Disdier P, Granel B, Serratrice J. et al. Cannabis arteritis revisited ten new case reports. Angiology 2001;52:505-506.

32. Lindsay $A C$, Foale RA, Warren O, Henry JA. Cannabis as a precipitant of cardiovascular emergencies. Int J Cardiol 2005;104: 230-232.

33. Pagotto U, Marsicano $S$, Cota D, Lutz B, Pasquali R. The emerging role of the endocannabinoid system in endocrine regulation and energy balance. Endocr Rev 2006;27:73-100.

34. Zuckerman B, Frank DA, Hingson R. et al.

Effects

of maternal marijuana and cocaine use on fetal growth. N Engl J Med 1989;320: 762-768.

35. Bernard C, Milh M, Morozov YM, Ben-Ari Y, Freund TF, Gozlan H. Altering cannabinoid signaling during development disrupts neuronal activity. Proc Nat Acad Sci USA 2005;102:9388-9393. 


\section{SUMMARY}

\section{Cannabis:}

\section{new anxiety}

Roger NORDMANN

Keywords

- alcohol

- cannabis

- drug
The regular using of cannabis by more than 1200000 french people is creating anxiety because of his noxious effects:

- great mental subordination;

- great somatic subordination;

- danger of his association with alcohol;

- incitation of using other drugs.

This using incitated the Académie Nationale de Médecine to edict recommandations. 